\title{
Implementation of a standardized robotic assistant surgical training curriculum
}

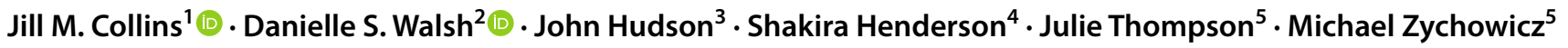

Received: 24 April 2021 / Accepted: 31 July 2021 / Published online: 26 August 2021

(c) The Author(s), under exclusive licence to Springer-Verlag London Ltd., part of Springer Nature 2021

\begin{abstract}
Since 2000, robotic-assisted surgery has rapidly expanded into almost every surgical sub-specialty. Despite the popularity of robotic surgery across the United States, a national consensus for standardized training and education of robotic surgeons or surgical teams remains absent. In this quality improvement initiative, a novel, stepwise iterative Robotic Assistant Surgical Training $(R A S T)$ curriculum was developed to broaden and standardize robotic bedside assistant training. Thirteen voluntary participants, capable of fulfilling the bedside assistant role, were evaluated to determine if RAST enhanced the learner's self-perceived level of confidence and comfort in their role as bedside assistant. A pre- and post-RAST training survey and a between-stages repeated-measures survey were conducted. All learner participants reported statistically significant increases in confidence and comfort after RAST training, $(p=<0.001)$, and between each stage, $F\left(2,24=60.47, p<.001 ; \eta_{\mathrm{p}}^{2}=0.834\right)$. Participant feedback regarding curriculum improvement was obtained, suggesting the desire for more training and practice, in smaller groups of 2-3 participants. One hundred percent of participants felt RAST was beneficial and that it should be implemented as standardized training during onboarding for all robotic bedside assistants. Thus, a standardized, stepwise iterative robotic bedside assistant curriculum increases learner preparedness, comfort, and confidence, safely away from the patient bedside.
\end{abstract}

Keywords Robotics · Robotic surgery $\cdot$ Robotic training $\cdot$ Quality $\cdot$ Robotic bedside assistant $\cdot$ Robotic-assisted surgery $\cdot$ Education

Jill M. Collins

Collinsji20@ecu.edu; jmc147@duke.edu

Danielle S. Walsh

walshd@ecu.edu

John Hudson

john.hudson@unchealth.unc.edu

Shakira Henderson

shakira.henderson@unchealth.unc.edu

Julie Thompson

julie.thompson@duke.edu

Michael Zychowicz

michael.zychowicz@duke.edu

1 Vidant Medical Center, 2100 Stantonsburg Road, Greenville, NC 27834, USA

2 East Carolina University, Greenville, NC, USA

3 University of North Carolina, Chapel Hill, NC, USA

4 UNC Health \& UNC School of Medicine, Chapel Hill, NC, USA

5 Duke University School of Nursing, Durham, NC, USA

\section{Introduction}

Since the U.S. Food and Drug Administration's (FDA) approval of the da Vinci robot, by Intuitive Surgical, Inc., in 2000 , robotic-assisted surgery (RAS) has substantially transformed minimally invasive surgical techniques [1]. Between 2008 and 2013, the utilization of RAS in the United States increased by $250 \%$ [2]. In 2015, an international group of robotic surgical experts and educators developed a standardized curriculum for robotic surgeons, known as the Fundamentals of Robotic Surgery (FRS). This educational platform is still undergoing validation through the Institute for Surgical Excellence [3, 4]. And despite the rapid adoption of RAS, healthcare professionals have yet to develop or implement a validated curriculum for the robotic surgeon or the surgical team.

It is well known that surgical team collaboration, competence, experience, and proficiency all contribute to optimal patient outcomes [5]. The role of the robotic bedside assistant (RBA) that was previously reserved for surgeons 
or surgical residents is now largely filled by mid-level providers, nurses, and surgical technicians. The physical separation of the surgeon from the patient and the RBA during the operation significantly increases the autonomous operative role of the RBA. The RBA facilitates operative efficiency, controls blood loss, manipulates tissue for exposure, places trocars, removes specimens and needles from the operative cavity, manually staples or sutures tissue, and clamps or compresses vessels [6-8]. All of these RBA responsibilities may directly impact the morbidity and mortality of robotically treated patients [5-9]. In addition, non-technical skills, such as communication, teamwork, leadership, and situational awareness, all contribute to hospital outcomes. Up to $70 \%$ of these adverse events are due to human error [10].

In a retrospective article by Leggett et al., overall robotic case length and blood loss were compared between non-surgeon and surgeon RBAs [9]. Over fourteen years, the study concluded that robotic console time with a non-surgeon RBA averaged 19.2 min faster than cases with a surgeon RBA, (95\% CI $0.26 \mathrm{~h}-0.37 \mathrm{~h}$ faster, $p<0.001$ ). Estimated blood loss was also approximately $47.5 \mathrm{cc}$ less with a nonsurgeon RBA (95\% CI 38.8-56.3 cc lower EBL, $p<0.001$ ).

There is very little information available that focuses on the training of the RBA. Intuitive Surgical, Inc., offers an online introductory training course, and an in-person first assistant course at limited facilities across the United States. However, Intuitive Surgical first assistant course includes facility fees, travel costs, and is provided based on availability. Cost constraints or competing robotic systems not manufactured by Intuitive Surgical, Inc., limit the applicability of this course to all end users. Often, as a result of limited resources and educational opportunities, informal training and education of the RBA is commenced at the patient bedside during the performance of patient operations.

Therefore, this quality improvement (QI) study was initiated to standardize the RBA training, via a three-stage stepwise iterative curriculum, known as Robotic Assistant Surgical Training (RAST). Robotic Assistant Surgical Training was developed to enhance the learner's self-perceived level of confidence and comfort as the RBA before direct-patient contact. In specific, the RBA learner's level of confidence and comfort in the domains of didactic, cognitive, psychomotor, fundamental knowledge, and team communication were evaluated across time. It was hypothesized that each stage of RAST would increase the learner's level of selfperceived confidence and comfort. To facilitate improvements in future versions of RAST, learners were asked to identify what aspects of the course were most helpful and to provide constructive critiques for improvements in the RAST curriculum. Finally, for internal program sustainability, learners were asked if they felt the RAST curriculum was beneficial to their role as RBA, and if they recommended or endorsed the curriculum as standard onboarding education and training.

\section{Methods}

Robotic Assistant Surgical Training is a three-stage, sequential stepwise curriculum implemented at a rural tertiary care hospital affiliated with an academic university. The QI study was conducted within the institution's robotics training lab. The participants had access to both Xi and Si da Vinci robots. Informed consent was obtained by all participants. Project approval was granted through Vidant Health's Center for Research and Grants. An institutional review board approval was not required since it met the institution's Quality Assurance/Quality Improvement project criteria.

A convenience sample of thirteen $(N=13)$ health care professionals eligible to fulfill the role of RBA from within the institution were included. Participation was voluntary and/or encouraged as part of the medical student or resident surgical education curriculum. Baseline participant demographic and professional experience data were obtained.

Inclusion criteria required that all participants held an active healthcare license that meets the criteria for any professional that can assume the role of the RBA, [Surgeon, M.D. or D.O., Fellow, M.D. or D.O., Resident, M.D. or D.O., Medical Intern, M.D., Medical Student, or D.O., Doctorate of Nursing practice (DNP), Certified Nurse Practitioner, (NP), Registered Nurse, (RN), Registered Nurse First Assistant, (RNFA), Physician's Assistant, (PA), Certified Surgical Technologist (CST), Foreign Medical Graduate, Surgical Assistant-Certified, (SA-C)]. Only healthcare employees or medical students affiliated with an academic program were considered. This included surgical residents required to participate as a part of their robotic training residency curriculum, or an elective rotation for medical students interested in a surgical residency. Additional inclusion criteria were participants having formal training in sterile technique or aseptic technique as well as completion of mandatory Stage I online training.

The three stages of RAST were delivered sequentially at three different time points (Table 1). In total, each session lasted approximately $2 \mathrm{~h}$ and took a total of 6 days to complete. Stage I: Introduction to Robotic Surgery, Stage II: Didactic Training Lab (Xi/Si platforms), and Stage III: Cognitive and Psychomotor Training with Team Communication. Stage I: Introduction to Robotic Surgery, is the current requirement for all RBA at the medical institution and includes registration to the online da Vinci community, hosted by Intuitive Surgical, Inc and completion of the first assistant introductory courses [11]. For this project, participant learners were instructed to complete both the first assistant $\mathrm{Xi}$ and Si modules that correspond to 'general surgery'. 
Table 1 Robotic assistant surgical training (RAST) curriculum

Preliminary RAST course survey

Objectives:

Evaluate participant experience and background

Obtain baseline participant comfort and confidence in robotic bedside assistant skills and needs

Stage I: introduction to robotic surgery

(a) Registration to da Vinci Surgery Online Community https://www.davincisurgerycommunity.com/

(b) Completion of online training and assessment modules

(c) Print and bring certificate of completion to day 1 Training Lab

Stage I: Between-stage effectiveness survey

Objectives:

Evaluate participant comfort and confidence in robotic bedside assistant skills and needs

Subjective participant feedback

Stage II: didactic training lab

(a) Orientation to the da Vinci robot and system components with interactive video tutorial and hands-on practical skills to include:

i. Docking of the da Vinci robot

$\mathrm{Si} / \mathrm{Xi}$

ii. Trocar and robotic port placement for docking

iii. Targeting of operative anatomy

iv. Instrument insertion and removal

v. Undocking of da Vinci robot

Stage II: between-stage effectiveness survey

Objectives:

Evaluate participant comfort and confidence in robotic bedside assistant skills and needs

Subjective participant feedback

Stage III: Cognitive and psychomotor training with team communication

(a) Educational lecture and review of robotic surgical operations led by an experienced bedside assistant providing:

i. Review of anatomy and physiology

ii. Surgery-specific patient positioning

iii. Surgery-specific port placement

iv. Instruction, explanation, and demonstration of the basic principles of RAS

v. Participant impromptu questions and feedback

(b) Non-technical skills seminar: team-training and communication techniques

(c) Simulation dry-lab: skills practice

Port Placement

Docking and targeting

Instrumentation Insertion and removal

Suture passing, cutting, following and removal

Bimanual laparoscopic skills and dexterity practice: Dual assistant ports

Team communication

Undocking

Stage III: between-stage effectiveness survey

Objectives:

Evaluate participant comfort and confidence in robotic bedside assistant skills and needs

Subjective participant feedback

Post-RAST course completion survey

Objectives:

Evaluate participant comfort and confidence in robotic bedside assistant skills and needs

Certificate of RAST completion signed off by PI and surgeon lead 
Stage II and Stage III are the projects' innovations. Fundamental, cognitive, psychomotor, didactic, and team communication robotic skills were incorporated in both stages. The stages were designed to reinforce, repeat, and build on the previous stage's knowledge and skills [12]. Both stages took approximately two hours to complete in groups of 2-10 participants based on lab and participant availability.

\section{Survey and data collection}

Two novel surveys were administered to participants during the study. The pre- and post-RAST survey was administered at the completion of Stage I (the current RBA training requirement) and at the completion of Stage III. The pre-/ post-survey consists of 25 questions that assess baseline comfort and confidence in cognitive, fundamental, psychomotor, didactic, and team communication RBA skills (Supplemental Table 1). The pre-survey includes baseline demographics. Both comfort and confidence are measured using a 5-point Likert scale, where $0=$ (Novice) No Confidence or No Comfort, $1=$ Uncomfortable or Unconfident, $2=$ Slightly Uncomfortable or Slightly Unconfident, $3=$ Comfortable or Confident, $4=$ Very Comfortable or Very Confident, $5=($ Expert $)$ Extremely Comfortable or Extremely Confident. At the completion of the post-RAST survey, learner participants were asked if they feel this curriculum was beneficial and if it should be implemented for all new RBA at VMC during onboarding.

Fundamental knowledge questions were drawn from the Intuitive Surgical, Inc., online community modules (Stage I), [11]. Didactic and psychomotor skills from Stage I were expanded into questions that evaluated participant confidence of performance in Stage II and III. Cognitive questions were derived to identify participant baseline knowledge of anatomical or surgical landmarks. Team training is explained in the online Intuitive Surgical, Inc., modules, and questions were developed to assess participant understanding of how communication between the RBA and surgeon is specific to robotic surgery. All questions were designed and incorporated into the pre- /post-survey by the project lead because they are directly applicable to what is currently being taught at the patient bedside to all novice RBAs.

A second novel RAST survey, the between-stages effectiveness (BSE) survey, was administered after each individual stage of RAST (Stages I-III). The BSE survey was created to emulate the Simulation Effectiveness Scale (SES) utilized by Sprehe et al. in the American Heart Association's Mega Code training [12]. In the SES learners were asked to report their perceived level of self-confidence after receiving repetitive simulation and training to master a skill set. Both the SES and the RAST BSE are designed to evaluate the impact that repetitive skills learning has on participant learners' confidence (Supplemental Table 2). Unlike the SES, the
RAST BSE survey consists of 11 questions that pertain to the domains assessed in the pre-/post-survey. Participants self-assessed their perceived level of confidence and comfort using the same 5-point Likert scale described above. In addition, two open-ended questions are asked to identify the strengths of each stage and areas for improvement for each stage supporting curriculum internal sustainability and continual improvement.

The BSE survey will identify if the individual stages of the curriculum were impactful to learner confidence or comfort, will identify which aspects of each stage were most beneficial to participant confidence or comfort, and what aspects of the stage could be improved upon. Trending survey results will identify whether or not a correlation exists between stage completion and the level of participant comfort or confidence as the RBA.

\section{Statistical analysis}

Data were analyzed using IBM SPSS version 27 software. Alpha was set at 0.05 . Shapiro-Wilk tests were conducted to examine the normality of the paired differences in both the pre/post-survey and the BSE survey, this included examining the difference in scores across each stage (after Stage I, after Stage II, and after Stage III). The mean sum of scores was compared for items regarding (1) fundamental, (2) cognitive knowledge, (3) psychomotor, (4) didactic robotic skills, and (5) team communication in separate repeated-measures analysis of variance (R-ANOVA).

To evaluate the impact of each stage (Stages I, II, III) of the RAST curriculum on learner's self-reported confidence and comfort, total scores for confidence/comfort were averaged for each participant and the scores were compared across the three data collection periods using a one-way analysis of variance (ANOVA). Paired t-tests were conducted to compare comfort and confidence in skills at pre- and post-RAST education. Themes from open-ended questions are presented in narrative format.

\section{Results}

\section{Participant demographics}

Study participant demographics are displayed in Table 2 . The most common professional level of training was resident (46.2\%). Males (53.8\%) and females (46.2\%) were equally represented. Eight (61.5\%) reported laparoscopic experience, and seven (53.8\%) reported robotic experience. All but two participants had experience of $<10$ robotic cases (84.6\%). The mean age was 32.08 years (range $26-58$ years). 
Table 2 Participant demographics

\begin{tabular}{lc}
\hline Variable & Data values \\
\hline Professional title, Number, $\%)$ & \\
Medical student & $5(38.5)$ \\
Nurse & $1(7.7)$ \\
Resident & $6(46.2)$ \\
Surgeon & $1(7.7)$ \\
Gender, Number, $(\%)$ & \\
Male & $7(53.8)$ \\
Female & $6(46.2)$ \\
Laparoscopic Experience, Number, $(\%)$ & $8(61.5)$ \\
Robotic Experience, Number, $(\%)$ & $7(53.8)$ \\
Robotic Cases, Years, $(\%)$ & \\
$0-5$ & $6(46.2)$ \\
$5-10$ & $5(38.5)$ \\
$10-20$ & $1(7.7)$ \\
$>50$ & $1(7.7)$ \\
Si, Number, $\%)$ & $2(15.4)$ \\
Xi, Number, $(\%)$ & $7(53.8)$ \\
Experience, Number, $(\%)$ & \\
0 & $3(23.1)$ \\
0.5 & $5(38.5)$ \\
1 & $1(7.7)$ \\
3 & $2(15.4)$ \\
Age in years, Mean (SD) & $2(15.4)$ \\
\hline
\end{tabular}

\section{Pre-/Post-RAST comfort and confidence survey}

The score was calculated for each skill at pre and post education utilizing the RAST curriculum. For all skills except Cognitive $(p=0.023)$ and Psychomotor $(p=0.021)$, the differences in the sum of scores were normally distributed and no extreme outliers were observed for any of the five skills. Results for the mean confidence/comfort scores at pre- and post-intervention for each skill are shown in Table 3. There was a statistically significant improvement in comfort and confidence with all skills scores and the total score. All participants $(100 \%)$ reported that the RAST curriculum was beneficial to the RBA. All participants (100\%) recommended the RAST curriculum and should be implemented as standardized training for all onboarding robotic bedside assistants at the institution.

\section{RAST between-stage effectiveness (BSE) survey}

The scores across the 11 Likert-scale items were summed for each participant with a possible total score ranging from 0 to 55 at Stage I, Stage II, and Stage III. The scores were showed normal distributions for paired differences. The overall test showed a statistically significant difference between stages, $F\left(2,24=60.47, p<0.001 ; \eta_{\mathrm{p}}^{2}=0.834\right)$. Post hoc pairwise revealed significant improvement from Stage I to II $(p<0.001)$; Stage II to III $(p<0.001)$; and Stage I to III $(p<0.001)$. Table 4 displays the mean BSE sum scores at each Stage.

Between-stage effectiveness themes.

Participants were asked to comment on two RBA elements they found helpful and well taught and two suggestions for curriculum improvement after each stage. Feedback was collected for both Stage II and Stage III. Common themes for topics well covered during Stage II skills included: "Docking and undocking," "How to load instruments," "Manipulating the instruments," "How to position arms...how to move boom," "Docking/ passing suture," and "How to set up and tear down pre/post-op." Suggestions for improvements to Stage II were: "Smaller groups, more time to practice," "Patient positioning and port placement," "Would like to see a full demonstration for draping, docking, etc.," and "Process of troubleshooting faults."

Common themes for Stage III participant positive feedback included: "Proper port placement," "Difference between Xi and Si console," Patient approach...location of ports for different procedures," "Table motion," "Additional methods of moving the arms/boom," "Ways to target the
Table 3 Skill comparison and pre- and post-intervention $(N=13)$

\begin{tabular}{lrrrrrrrr}
\hline Skill & \multicolumn{1}{l}{ Pre } & & & \multicolumn{2}{l}{ Post } & & $t$-value & $p$-value \\
\cline { 2 - 3 } & Mean & \multicolumn{1}{c}{ SD } & & Mean & SD & & \\
\hline Fundamental-8 items & 6.61 & 9.31 & & 25.00 & 6.46 & -7.76 & $<0.001$ \\
Cognitive-3 items & 2.69 & 2.53 & 9.61 & 2.50 & -8.88 & $<0.001$ \\
Psychomotor-10 items & 9.08 & 10.48 & & 31.69 & 7.49 & -7.82 & $<0.001$ \\
Didactic-3 items & 1.46 & 2.11 & & 10.00 & 3.06 & -9.31 & $<0.001$ \\
Team Communication-1 item & 1.46 & 1.45 & & 3.59 & 1.05 & -5.42 & $<0.001$ \\
Total Score-25 items & 21.31 & 21.93 & & 79.85 & 18.17 & -8.69 & $<0.001$ \\
\hline
\end{tabular}

Response scale is $0=$ (Novice) No confidence or no comfort, $1=$ Uncomfortable or unconfident, $2=$ Slightly uncomfortable or slightly unconfident, $3=$ Comfortable or confident, $4=$ Very comfortable or very confident, $5=$ (Expert) extremely comfortable or extremely confident 
Table 4 BSE mean score at Stage 1 , stage 2 , and stage 3 $(N=13)$

\begin{tabular}{|c|c|c|c|c|c|c|}
\hline \multirow[t]{2}{*}{ Variable } & \multicolumn{2}{|c|}{ Stage 1} & \multicolumn{2}{|c|}{ Stage 2} & \multicolumn{2}{|c|}{ Stage 3} \\
\hline & Mean & SD & Mean & SD & Mean & SD \\
\hline BSE Survey Sum Score ${ }^{a}$ & 8.31 & 10.05 & 27.0 & 1.63 & 39.23 & 8.56 \\
\hline
\end{tabular}

$B S E$ between-Stages Effectiveness

${ }^{\text {a }}$ Score is calculated as the sum of responses on 11 items with possible range from 0 to 55 . Response choices include $* 0=$ (Novice) No Confidence or No Comfort, $1=$ Uncomfortable or unconfident, $2=$ Slightly uncomfortable or slightly unconfident, $3=$ Comfortable or Confident, $4=$ Very comfortable or very confident, $5=$ (Expert) Extremely comfortable or extremely confident robot," "How to arrange Xi arms to avoid collisions" Suggestions for Stage III improvements were: "Longer training?," "More console with exercises," "More practice driving the robot," "More practice operating with the robot," "Second review," and "Brief video discussing differences between Si and Xi."

Common themes of areas that the participants thought well covered will continue for the next iteration of RAST. The commonly identified constructive critiques such as smaller groups with more hands-on time as well as several full complete demonstrations. These suggestions will be integrated in RAST to meet learner RBA needs.

\section{Discussion}

Since 2011, many articles have been published analyzing the training modalities used for robotic surgeons [13]. Common training techniques include didactic education, skills labs, virtual reality simulation, cadavers, case observation, proctoring, mentoring, and assessment [14]. FRS may be the most well-described international curriculum within robotic literature. However, as mentioned previously, FRS is still undergoing assessment and validation through the Department of Defense and the Institute for Surgical Excellence $[3,15]$. The FRS curriculum is composed of four modules: (1) Introduction to da Vinci Surgical Systems, (2) Didactic training, (3) Psychomotor skills development, and (4) Team training with an emphasis on communicative skills [3]. However, FRS is surgeon specific and not team specific. FRS skills training requires access to an integrated virtual reality simulation system. This limits organizations with financial constraints that may not be able to purchase robotic simulators.

Robotic skills improvement can be achieved without FRS virtual reality simulation through stepwise iterative approaches including didactic modules, skills labs with or without models, implementing a required minimum number of RBA cases, then finally proctoring $[13,14]$. The RAST curriculum was designed to incorporate specific FRS module themes, but through educational modalities that are obtainable for all organizations and with a focus on the RBA. Recent studies have shown that using a stepwise approach, whether via an inanimate model or via the virtual reality FRS da Vinci skills simulator (dVSS), equally improves surgeon clinical skills $(p=0.833, p<0.001$; $p=0.805, p<0.001$ respectively), [16]. Regardless of the training modality used, a systematic, stepwise training curriculum with procedure-based competencies consistently increases the robotic surgeon's confidence and proficiency [13-17].

As mentioned previously, the RBA is an important component of the robotic surgery team [9]. The art of assisting is a skillset in its own right, and it is a role that surgeons have performed throughout their own robotic education and training. Recently, in a study by Turner [17] the majority of surgeons, $(n=20,80 \%)$ and fellows $(n=11,55 \%)$ polled at Memorial Sloan Kettering Cancer Center, felt that the most optimal way to learn how to train for robotic surgery was to master the role of RBA from a 'skilled' RBA.

Turner [17] also identified that RBA confidence and comfort develops through a significant learning curve, $F$ $\left(2,24=60.47, p<0.001 ; \eta_{\mathrm{p}}^{2}=0.834\right)$. This is consistent with studies that show a significant learning curve for the robotic surgeon [18, 19]. In 2021, Raison et al. [18] took thirty-nine participants, of similar professional backgrounds compared to our study, through a live-porcine training course that included basic RBA skills. Participants rated their pre-training confidence low $(3.5 \pm 7.4)$, while perceiving their post-intervention technical skills to have significantly increased from baseline $(p<0.0001)$, [18]. In addition, participants found that the basic RBA fundamental skills (docking, positioning, port placement) were beneficial to all learners [18]. The results are comparable to our own, illustrating the importance of developing training modalities outside the operating room that decrease anxiety and increase RBA confidence and knowledge.

In 2014, a similar stepwise training modality to FRS was created known as the Robotic Training Network (RTN) [20]. The RTN was initially developed to standardize the training of obstetrics and gynecology and/or 
general surgical residents or fellows [20,21]. As a result of the RTN, a validated didactic skills checklist, known as the Robotic-Objective Structured Assessments of Technical Skills (R-OSATS), was founded. Surgical residents or fellows are graded on five didactic console skills using R-OSATS by expert robotic surgeons. Through R-OSATS, Siddiqui et al. [21] were able to show that trainee/learner performance outcomes were significantly impacted by previous surgical or robotic surgical experience. Faculty outperformed fellows, $(p<0.01)$, and fellows outperformed residents, $(p<0.01),[21]$. Although these results were statistically significant, it is unclear the degree that expert bias may have impacted trainee/learner R-OSATS performance ratings across healthcare systems.

Currently, the RTN is supported by the Accreditation Council for Graduate Medical Education and, like FRS, the Institute for Surgical Excellence [20, 21]. However, neither program focuses on the RBA role or the impact of RBA confidence or comfort on the performance of R-OSATS or GEARS skills. Further, it is unclear if the accrediting bodies plan to integrate the R-OSATS didactic assessment or the virtual reality GEARS assessment into a universal curriculum for robotic surgical trainees. Regardless, both the RTN and FRS curriculums support that a stepwise curriculum can improve robotic surgical performance, similar to the RAST program.

The lack of a standardized effective educational curriculum for the RBA has probably hampered the effectiveness of the robotic surgery team. TeamSTEPPS, an evidence-based framework for improving quality care and patient outcomes, emphasizes the importance of cultivating team attitudes, to increase knowledge, performance, and skills [22]. Souders et al. [23] reviewed 24 robotic Sacrocolpopexy cases and identified how any human factor can impact or disrupt surgical flow. Docking and port placement, roles typically assumed by the RBA, accounted for the highest rate of disruptions to surgical flow, $(19.2 \pm 14.4$ flow disruptions per hour), [22]. Training, (24\%), or any interruption caused by lack of skill or knowledge by the RBA, was identified as the factor most responsible for surgical interruptions [22].

In 2015, Potretzke, et al. [6] compared "junior" level assistants to "senior" level assistants over the span of 3 years, concluding that assistant experience had no impact on operative blood loss $(p=0.656)$, post-operative complications $(p=0.916)$, or blood loss $(p=0.488)$. In contrast, Leggett et al. determined that a well-trained RBA can significantly impact time and estimated surgical blood loss [9].

Participant feedback is instrumental for program sustainability. Subjective feedback ensures that the curriculum meets the demands of the RBA learner's needs. The majority of participants felt that this training was important to their role by voicing the need for more training, in smaller focused groups with more hands-on time.
Participant critique of the program encourages internal program development, learner specific integration, and sustainability within individual institutions.

The strengths of our QI study are that the results are comparable to similar stepwise robotic curriculums. The framework of RAST is therefore validated when compared to the process of FRS and non-virtual reality stepwise robotic surgeon training. The advantage of RAST is that it is specifically designed for the RBA role and is applicable and helpful to all learners. This study also showed that surgeons and nurses, regardless of previous experience, benefited from RAST in building their confidence in RBA skills across the five domains of cognitive, psychomotor, didactic, team communication, and fundamental knowledge. Last, it is evident that the need to evaluate the learning curve for the RBA should be further examined.

The RAST curriculum is easily adaptable and transferable to other institutions. Although the authors had a robotics training lab on site, the study could be done in a conventional operating room provided there is access to a robot surgical platform. Further, the RAST stepwise curriculum may be transferrable to other robotic platforms.

Limitations should be considered. The course was only offered within a single institution. Survey-specific questions may not be transferable across organizations based on robotic equipment and brand. Selection bias may skew results since there was not a random selection of participants and participants were grouped with peers. Second, there is little comparison data or literature specific to the RBA and most comparative literature is specific to surgeon training only. Self-reported comfort and confidence may have also skewed results, however this bias may be minimal due to the normal distribution of skill score increases across the three stages of training for all participants. Costefficiency of training was not analyzed since access, time, and educator availability were the primary constraints to this project. The study was postponed several times due to the COVID-19 pandemic and restrictions on group education and training narrowing the timeframe to complete the study. The study originally called for 30 participants but due to the above barriers the study capped at 13 participants. However, statistical results were so compelling with this pilot group that additional trainees were unlikely to alter the conclusions.

There are several future directions that the authors would propose. Task, or time-to-task, training can be measured and these data could further validate the RAST curriculum. Integration of surgery-specific video tutorials with focus on anatomical landmarks from robotic procedures would prepare the RBA more specifically for certain operations. Adding a Stage IV cadaver model would potentially permit a more immersive interactive-team training to strengthen team communication and operative technique. 


\section{Conclusions}

The importance of the RBA role has been well described but the training and education has failed to become a priority. As the surgeon workforce is predicted to diminish over the next decade, mid-level providers who fulfill the role of the RBA in surgery will become more significant [24]. Already, the role of the RBA, which was historically reserved for surgeons or surgical residents, is largely filled by mid-level providers, nurses, and surgical technicians. Thus, training of the RBA becomes even more critical as surgeons rely on assistance from healthcare workers that have not been trained or educated to the same degree as surgeons.

Based on the findings of this study, the manufacturercreated Stage I online training module can be improved with the addition of RAST. The stepwise, iterative RBA directed curriculum of RAST significantly improved learner's comfort and confidence in their fundamental, cognitive, didactic, psychomotor, and team communication skills. Each Stage of RAST made a statistically significant impact on the learner's self-perceived level of confidence and comfort. Further work to validate the effectiveness of RAST will allow it to serve as a foundation for standardized RBA training within nursing, physician, and possibly surgical technician educational programs.

Supplementary Information The online version contains supplementary material available at https://doi.org/10.1007/s11701-021-01291-8.

Acknowledgements David A. Rodeberg, MD, Editor. Homayoun Pournik, MD, RAST educator, Stage II-III.

Funding This quality improvement initiative did not receive any specific grant from funding agencies in the public, commercial, or notfor-profit sectors. No funding was received for manuscript preparation.

\section{Declarations}

Conflict of interest Author Jill M. Collins, author Danielle S. Walsh, author Shakira Henderson, author John Hudson, author Julie Thompson, and author Michael Zychowicz declare that they have no conflict(s) of interest.

Ethics approval Not required since this is a QI project.

Informed consent All procedures followed were in accordance with the ethical standards of the responsible committee on human experimentation (institutional and national) and with the Helsinki Declaration of 1975 , as revised in 2000. Informed consent was obtained from all participants for being included in the study.

\section{References}

1. Julian D, Tanaka A, Mattingly P, Truong M, Perez M, Smith $R$ (2018) A comparative analysis and guide to virtual reality robotic surgical simulators. Int J Med Robot Comput Assist Surg 14(1):e1874. https://doi.org/10.1002/rcs.1874

2. Cundy T, Mayer E, Camps J, Olsen L, Pelizzo G, Yang GZ, Darzi A, Najmaldin A (2015) Education and training in pediatric robotic surgery: lessons learned from an inaugural multinational workshop. J Robot Surg 9(1):57-63

3. FRS: Fundamentals of Robotic Surgery (n.d.). https://frsurgery. org

4. Vetter M, Green I, Martino M, Fowler J, Salani R (2015) Incorporating resident/fellow training into a robotic surgery program. J Surg Oncol 112:684-689

5. Kumar A, Smith R, Patel V (2015) Current status of robotic simulators in acquisition of robotic surgical skills. Curr Opin Urol 25:168-174

6. Potretzke A, Knight B, Brockman J, Vetter J, Figenshau R, Bhayani S, Benway B (2016) The role of the assistant during robotassisted partial nephrectomy: does experience matter? J Robot Surg 10:129-134

7. Ramanathan R, Martinez Salamanca J, Mandhani A, Leung R, Rao S, Berryhill R, Tewari A (2009) Does 3-dimensional (3-D) visualization improve the quality of assistance during robotic radical prostatectomy? World J Urol 27:95-99

8. Sessa L, Sessa L, Perrenot C, Perrenot C, Xu S, Xu S, Hubert J, Hubert J, Bresler L, Bresler L, Brunaud L, Brunaud L, Perez M, Perez M (2018) Face and content validity of xperienceTM team trainer: bed-side assistant training simulator for robotic surgery. Updates Surg 70(1):113-119. https://doi.org/10.1007/s13304-0170509-x (2017)

9. Leggett LK, Muldoon O, Howard DL, Kowalski LD (2021) A comparison of surgical outcomes among robotic cases performed with an employed surgical assist versus a second surgeon as the assist. J Robot Surg. https://doi.org/10.1007/s11701-021-01230-7

10. Gjeraa K, Spanager L, Konge L, Petersen RH, Østergaard D (2016) Non-technical skills in minimally invasive surgery teams: asystematic review. Surg Endosc 30(12):5185-5199. https://doi. org/10.1007/s00464-016-4890

11. Intuitive Surgical, Inc. (2019) da Vinci surgery community. Intuitive Surgical Inc,

12. Sprehe J, March AL, Wilson CB, Park HS (2016) The effect of videoconferencing on code blue simulation training. Clin Simul Nurs 12(7):260-267. https://doi.org/10.1016/j.ecns.2016.02.013

13. Tennant K, Rivers C (2019) Sterile technique. StatPearls Publishing

14. Schreuder H, Wolswijk R, Zweemer R, Schijven M, Verheijen $R$ (2011) Training and learning robotic surgery, time for a more structured approach: a systematic review. BJOG Int J Obstet Gynecol 119:137-149

15. Raza SJ, Froghi S, Chowriappa A, Ahmed K, Field E, Stegemann AP, Rehman S, Sharif M, Shi Y, Wilding GE, Kesavadas TJ, Guru KA (2014) Construct validation of the key components of fundamental skills of robotic surgery (FSRS) curriculum-a multi-institution prospective study. JSurg Educ 71(3):316-324. https://doi.org/10.1016/j.jsurg.2013.10.00615

16. Aghazadeh MA, Mercado MA, Pan MM, Miles BJ, Goh AC (2016) Performance of robotic simulated skills tasks is positively associated with clinical robotic surgical performance. BJU Int 118(3):475-481. https://doi.org/10.1111/bju.13511

17. Turner SR, Mormando J, Park BJ, Huang J (2020) Attitudes of robotic surgery educators and learners: challenges, advantages, tips and tricks of teaching and learning robotic 
surgery. J Robot Surg 14(3):455-461. https://doi.org/10.1007/ s11701-019-01013-117

18. Raison N, Poulsen J, Abe T, Aydin A, Ahmed K, Dasgupta $P$ (2020) An evaluation of live porcine simulation training for robotic surgery. J Robot Surg. https://doi.org/10.1007/ s11701-020-01113-3

19. Schreuder HWR, Wolswijk R, Zweemer RP, Schijven MP, Verheijen RHM (2012) Training and learning robotic surgery, time for a more structured approach: a systematic review. BJOG Int J Obstet Gynaecol 119(2):137-149. https://doi.org/10.1111/j.1471-0528. 2011.03139.x

20. Polin MR, Siddiqui NY, Comstock BA, Hesham H, Brown C, Lendvay TS, Martino MA (2016) Crowdsourcing: a valid alternative to expert evaluation of robotic surgery skills. Am J Obstet Gynecol 215(5):644.e1-644.e7. https://doi.org/10.1016/j.ajog. 2016.06.033

21. Siddiqui N, Galloway M, Geller E, Green I, Hur H, Langston K, Pitter M, Tarr M, Martino M (2014) Validity and reliability of the robotic objective structured assessment of technical skills. Obstet Gynecol 123(6):1193-1199. https://doi.org/10.1097/AOG.00000 00000000288

22. Esce A, Rodeberg DA, Rothstein DH, Browne M, Wakeman D, American Academy of Pediatrics Section on Surgery's Delivery of Surgical Care Committee (2018) Prevalence and perceptions of team training programs for pediatric surgeons and anesthesiologists. J Surg Res 232:559-563. https://doi.org/10.1016/j.jss.2018. 07.025

23. Souders CP, Catchpole K, Hannemann A, Lyon R, Eilber KS, Bresee C, Cohen T, Weigl M, Anger JT (2019) Flow disruptions in robotic-assisted abdominal sacrocolpopexy: does robotic surgery introduce unforeseen challenges for gynecologic surgeons? Int Urogynecol J 30(12):2177-2182. https://doi.org/10.1007/ s00192-019-03929-6

24. National Center for Health Workforce Analysis (2016) National and regional projections of supply and demand for surgical specialty practitioners: 2013-2025. U.S. Department of Health and Human Services; Health Resources and Services Administration; Bureau of Health Workforce; National Center for Health Workforce Analysis

Publisher's Note Springer Nature remains neutral with regard to jurisdictional claims in published maps and institutional affiliations. 